\title{
Polyphenol-Based Design of Functional Olive Leaf Infusions ${ }^{\S}$
}

\section{Željka Peršurić\# $₫$, Lara \\ Saftić\# ${ }^{\oplus}$, Dora Klisović $\odot$ and \\ Sandra Kraljević Pavelić* \\ University of Rijeka, Department of Biotechnology, Centre for High- -Throughput Technologies, Radmile Matejčić 2, 51000 Rijeka, Croatia}

Received: 30 June 2018 Accepted: 22 May 2019

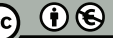

\footnotetext{
*Corresponding author:

Phone: +38551584550

E-mail: sandrakp@biotech.uniri.hr

\#These authors contributed equally

${ }^{\text {sPaper }}$ was presented at the 9 th International Congress of Food Technologists, Biotechnologists and Nutritionists, 3-5 October 2018, Zagreb, Croatia
}

\section{SUMMARY}

The beneficial properties of polyphenols are widely recognized, and polyphenol-rich olive oil, which is part of the typical Mediterranean diet, has been identified as having positive health effects. However, over the past decade, olive leaves have been discovered as an alternative polyphenol-rich source. This is particularly interesting in the context of the growing interest in functional foods, as well as in terms of the management of biological waste, including olive leaves that are left over from the production of olive oil. Previous studies on olive leaves confirmed that they have a high phenolic content, which explains their previously described strong antibacterial, antimicrobial and antiviral activity. Therefore, the major aim of our work is to comprehensively determine olive leaf phenolic content in cultivars Istarska bjelica, Leccino and Buža as a natural source of bioactive compounds suitable for daily consumption in the form of infusion. For this purpose, we examined the influence of olive leaf cultivar, maceration time and temperature on the phenolic composition of final infusions. Phenolic compounds were analysed by liquid chromatography (LC) coupled to a triple quadrupole mass spectrometer (LC-QQQ). As expected, the results indicate the significant influence of not only the olive cultivar but also of maceration parameters on the qualitative and quantitative phenolic composition. The highest phenolic compound content was obtained in the infusion of Istarska bjelica leaves after $15 \mathrm{~min}$ of maceration. However, the Buža olive leaf infusion had the most diverse phenolic composition. Furthermore, we designed several functional olive leaf infusion mixtures with phenolic compositions adjusted based on the desired health effect. The results show the role of phenolic composition adjustment in the development and improvement of the quality of functional olive leaf infusions.

Key words: olive leaf infusion, LC-QQQ, polyphenols, functional infusions

\section{INTRODUCTION}

Polyphenols, as plant secondary metabolites, show a high degree of structural diversity. They are present and stored in plant tissues in the form of different derivatives, mainly as sugar O-glycosides (1). Glycosylation ensures their structural stability during storage in the vacuoles and chloroplasts of plants.

Olive oil is considered to be outstanding due to its high polyphenolic content, and it is well-known for its nutritive and health-promoting potential (2). In addition to the olive fruit, from which olive oil is derived, olive leaves are also a rich source of phenolic compounds (3). However, olive leaves have not yet been sufficiently exploited in medical applications as a source of beneficial phenolics. Interestingly, olive leaves are traditionally trimmed from olive trees, but are not used afterwards, typically ending up as biological waste. The exploitation of olive leaves as a starting raw material in the production of functional food or other medical products may thus improve biological waste management.

Data from a number of studies suggest that olive leaf extracts reduce the risk of cardiovascular disease (CVD) due to their anti-atherosclerotic, hypotensive, antioxidant, anti-inflammatory and hypocholesterolaemic effects (4). The phenolic compounds from leaves also 
possess antimicrobial (2) and neuroprotective activity (5). Nevertheless, limited data on the effects of olive leaf polyphenols in humans is available in the scientific literature. Still, some randomized clinical studies show that olive leaf polyphenols are favourable modulators of blood pressure, plasma lipid profiles (4) and insulin sensitivity in overweight people (6).

The colorimetric Folin-Ciocalteu assay is the most used and rapid quantitative technique for determining the content of total polar phenolics. This method has been used previously for olive leaf analyses (7). However, colorimetric methods provide information only about total phenolic content, without giving any details about their characterization. In order to obtain a detailed representation of the phenolic composition of olive leaves, chromatographic methods are used in combination with various detectors. High-performance liquid chromatography (HPLC) is the most widely used method for analysing phenolic compounds. However, for a detailed determination of the phenolic composition, as well as all possible phenolic derivatives, mass spectrometry (MS) techniques are increasingly employed. MS provides higher selectivity and sensitivity than other methods and has been shown to be one of the most effective techniques in biomedical research, especially for the analysis of complex biological matrices (8).

Therefore, in this paper we determined the phenolic composition of three olive leaf cultivars (i.e. Istarska bjelica, Leccino and Buža) by liquid chromatography (LC) coupled to a triple quadrupole mass spectrometer (QQQ). The obtained data were used to design functional olive leaf infusions, because this form of preparation is suitable for daily consumption and has been accepted in many cultures. Additionally, water is a cheap and non-hazardous polar solvent. By using a mixture design approach, herein we propose several different mixtures of analysed olive leaf cultivars with the desired phenolic content assumed to yield a specific biological effect.

\section{MATERIALS AND METHODS}

\section{Reagents and chemicals}

The 2,5-dihydroxybenzoic acid (2,5-DHBA), 3,4-dihydroxybenzoic acid (3,4-DHBA), 4-hydroxybenzoic acid ( $p \mathrm{HBA})$, 3-hydroxytyrosol, apigenin, caffeic acid, chrysin, quercetin, naringenin, diosmetin, rutin, luteolin, luteolin-7-O-glucoside, p-coumaric acid, oleuropein and tyrosol were all from Sigma-Aldrich (Merck, St. Louis, MO, USA). Ferulic acid was purchased from Extrasynthese (Genay, France). Apigenin-7-O-glucoside and verbascoside were obtained from HWI Analytik GmbH (Rülzheim, Germany). Gallic acid was obtained from Alfa Aesar (Thermo Fisher Scientific, Waltham, MA, USA).

Chemical reagents methanol (LC-MS purity) and acetonitrile (LC-MS purity) were purchased from Honeywell Research Chemicals (Bucharest, Romania). Formic acid (LC-MS purity) was obtained from Sigma-Aldrich, Merck. Milli-Q water was obtained using a Milli-Q water purification system $(<0.058$ $\mu \mathrm{S} / \mathrm{cm}$, Mili-Q model Pacific TII 12; Barnstead ${ }^{\mathrm{TM}}$, Thermo Fisher Scientific).

\section{Olive leaf extraction procedures}

Olive leaves of Istarska bjelica, Leccino and Buža varieties were obtained from the same olive plantation (45⒈'53.6"N, $\left.13^{\circ} 35^{\prime} 04.8^{\prime \prime E}\right)$ in Novigrad, Istria, Croatia (April 2017). Samples were dried and stored in the dark at room temperature until analysis. Simulating household conditions, the olive leaf infusions were prepared from olive leaves roughly ground in a mortar. For optimization of extraction procedure, olive leaves from Istarska bjelica cultivar were used. In this process, $0.2 \mathrm{~g}$ of ground olive leaves were macerated in $20 \mathrm{~mL}$ of water that was heated to three different temperatures $\left(80,90\right.$ and $\left.100^{\circ} \mathrm{C}\right)$. Leaves were macerated in hot water for 1, 5, 10, 15 or 20 min. Organic extraction was carried out by adding $4 \mathrm{~mL}$ of methanol to $50 \mathrm{mg}$ of chopped leaves. Afterwards, samples were sonicated in an ultrasonic bath (Sonorex Digitec, Bandelin electronic GmbH \& Co. KG, Berlin, Germany) for 10 min at a constant temperature of $25^{\circ} \mathrm{C}$. All extracts were filtered using Chromafil ${ }^{\circledast}$ cellulose acetate microfilters $(0.45 \mu \mathrm{m}, 25 \mathrm{~mm}$; Macherey-Nagel $\mathrm{GmbH} \&$ Co. KG, Düren, Germany) before analysis.

\section{LC-QQQ analysis}

An Agilent 1260 series HPLC chromatograph equipped with a degasser, binary pump, auto-sampler and column oven coupled to an Agilent 6460 triple quadrupole mass spectrometer equipped with Jet Stream electrospray (AJS ESI) source was used for quantitative analysis and the data was processed with MassHunter workstation software v. B.07.00 (Agilent Technologies, Inc., Palo Alto, CA, USA).

For chromatographic separation of phenolic compounds, two methods were developed. In the first method, chromatographic analyses were carried out on Zorbax SB-C18, Rapid Resolution HT, $60000 \mathrm{kPa}$ column (2.1 mm i.d. $\times 50 \mathrm{~mm}$, particle size $1.8 \mu \mathrm{m}$; Agilent Technologies, Inc.). The mobile phase consisted of: $0.1 \%$ formic acid in Milli-Q water $(A)$ and $0.1 \%$ formic acid in acetonitrile (B). The gradient elution was modified as follows: 0-0.9 min linear gradient from 1 to $10 \% \mathrm{~B}, 0.9-3 \mathrm{~min}$ from 10 to $20 \%$ B, 3-4.5 min from 20 to $25 \%$ B, 4.5-6 min from 25 to $30 \%$ B, 6-7.5 min at $30 \%$ B, 7.5-9 min from 30 to $90 \%$ B, 9-9.30 min at $90 \%$ B, 9.30-9.60 min from 90 to $10 \% B$ and 9.60-15 min from 10 to $1 \% \mathrm{~B}$. Holding time was set to 2 min to equilibrate the system. The flow rate was $0.33 \mathrm{~mL} / \mathrm{min}$. The column temperature was maintained at $30^{\circ} \mathrm{C}$. The sample injection volume was 2.5 $\mu \mathrm{L}$. For MS, the parameters were set as follows: capillary voltage was $3.5 \mathrm{kV}$ in both positive and negative mode, nozzle voltage $0.5 \mathrm{kV}$, ion source temperature was set to $300^{\circ} \mathrm{C}$, gas flow was 5 $\mathrm{L} /$ min, nebulizer pressure $310 \mathrm{kPa}$, drying gas temperature 250 ${ }^{\circ} \mathrm{C}$ and sheath gas flow $11 \mathrm{~L} / \mathrm{min}$. Nitrogen was used as collision gas and collision energies were from 0 to $40 \mathrm{~V}$ (Table 1).

In the second method, tyrosol was analysed using the same chromatographic column and mobile phases, but with different gradient elution: 0-0.1 min linear gradient from 1 to $10 \%$ $\mathrm{B}, 0.1-13.39 \mathrm{~min}$ from 10 to $90 \% \mathrm{~B}, 12.39-14.59 \mathrm{~min}$ from 90 to $10 \% \mathrm{~B}$, from $14.59-16 \mathrm{~min}$ from 10 to $1 \% \mathrm{~B}$ and $16-20 \mathrm{~min}$ at 1 $\%$ B. Holding time was set to $4 \mathrm{~min}$. The flow rate was $0.1 \mathrm{~mL} /$ 
Table 1. Liquid chromatography coupled to a triple quadrupole mass spectrometer (LC-QQQ) parameters used for characterization of phenolic fractions

\begin{tabular}{|c|c|c|c|c|c|c|c|c|c|c|c|c|}
\hline Polyphenol standard & Mode & $\begin{array}{l}\text { Precursor } \\
\text { ion } \\
(\mathrm{m} / \mathrm{z})\end{array}$ & $\frac{\text { Frag }}{\mathrm{V}}$ & $\begin{array}{c}\text { Product } \\
\text { ion }(m / z)\end{array}$ & $\frac{C E}{V}$ & Slope (a) & $\begin{array}{l}\text { Intercept } \\
\text { (b) }\end{array}$ & $\begin{array}{l}\text { Linearity } \\
\text { range } \\
\mu \mathrm{g} / \mathrm{mL}\end{array}$ & $\mathrm{R}^{2}$ & $\frac{\mathrm{LOD}}{\mu \mathrm{g} / \mathrm{mL}}$ & $\frac{\mathrm{LOQ}}{\mu \mathrm{g} / \mathrm{mL}}$ & $\frac{\mathrm{RT}}{\min }$ \\
\hline 2,5-DHBA & - & 152.8 & 80 & $\begin{array}{c}108.0^{*} \\
81.8 \\
53.0\end{array}$ & $\begin{array}{l}20 \\
16 \\
20\end{array}$ & 15806.7 & 33.47 & $0.001-7.5$ & 0.995 & 0.007 & 0.021 & 4.42 \\
\hline 3,4-DHBA & - & 152.9 & 90 & $\begin{array}{c}108.0^{*} \\
81.0 \\
53.0\end{array}$ & $\begin{array}{l}20 \\
16 \\
18\end{array}$ & 3761.5 & 15.01 & $0.005-7.5$ & 0.998 & 0.013 & 0.040 & 1.70 \\
\hline 3-Hydroxytyrosol & - & 152.9 & 100 & $\begin{array}{c}95.0^{*} \\
94.8\end{array}$ & $\begin{array}{l}18 \\
20\end{array}$ & 756.9 & 18.41 & $0.1-2.5$ & 0.994 & 0.080 & 0.243 & 1.73 \\
\hline Apigenin & - & 268.9 & 160 & $\begin{array}{c}149.0 \\
116.9^{*}\end{array}$ & $\begin{array}{l}24 \\
22\end{array}$ & 41665.0 & 7.67 & $0.001-0.5$ & 0.995 & 0.001 & 0.002 & 10.33 \\
\hline Apigenin-7-O-glucoside & - & 430.9 & 200 & $\begin{array}{c}310.9 \\
268.1^{*}\end{array}$ & $\begin{array}{l}24 \\
36\end{array}$ & 90425.9 & -7.26 & $0.001-0.5$ & 0.997 & 0.000 & 0.001 & 7.55 \\
\hline Caffeic acid & - & 178.8 & 100 & $\begin{array}{c}135.0^{*} \\
116.9 \\
88.9\end{array}$ & $\begin{array}{l}12 \\
24 \\
34\end{array}$ & 27277.2 & 206.79 & $0.005-5$ & 0.991 & 0.025 & 0.076 & 5.35 \\
\hline Chrysin & + & 254.8 & 170 & $\begin{array}{c}153.3^{*} \\
103.4 \\
69.3\end{array}$ & $\begin{array}{l}30 \\
36 \\
50\end{array}$ & 14654.97 & 92.72 & $0.005-7.5$ & 0.996 & 0.021 & 0.063 & 11.159 \\
\hline Ferulic acid & - & 192.9 & 100 & $\begin{array}{c}177.9 \\
149.0 \\
134.0^{*}\end{array}$ & $\begin{array}{c}8 \\
6 \\
12\end{array}$ & 2289.7 & 4.91 & $0.01-10$ & 0.997 & 0.007 & 0.021 & 6.70 \\
\hline Gallic acid & - & 168.8 & 100 & $\begin{array}{c}125.0^{*} \\
78.9\end{array}$ & $\begin{array}{l}10 \\
20\end{array}$ & 11611.28 & 58.78 & $0.01-7.5$ & 0.998 & 0.0167 & 0.0506 & 0.856 \\
\hline Luteolin & - & 284.9 & 180 & $\begin{array}{c}150.9 \\
133.0^{*}\end{array}$ & $\begin{array}{l}24 \\
36\end{array}$ & 26778.7 & 63.89 & $0.001-5$ & 0.994 & 0.008 & 0.024 & 9.11 \\
\hline Naringenin & - & 270.9 & 140 & $\begin{array}{c}151.0^{*} \\
118.9\end{array}$ & $\begin{array}{l}12 \\
24\end{array}$ & 33448.0 & 10.74 & $0.001-0.5$ & 0.998 & 0.001 & 0.003 & 10.16 \\
\hline Oleuropein & - & 538.9 & 170 & $\begin{array}{c}377.3 \\
307.3 \\
275.1^{*}\end{array}$ & $\begin{array}{l}12 \\
18 \\
18\end{array}$ & 15681.8 & -10.76 & $0.001-2.5$ & 0.991 & 0.002 & 0.007 & 8.07 \\
\hline$p$-coumaric acid & - & 162.9 & 90 & $\begin{array}{c}119.0^{*} \\
92.8 \\
64.9\end{array}$ & $\begin{array}{l}12 \\
36 \\
48\end{array}$ & 30897.4 & 769.42 & $0.025-2.5$ & 0.991 & 0.082 & 0.249 & 6.26 \\
\hline$p \mathrm{HBA}$ & - & 136.9 & 80 & $\begin{array}{l}93.0^{*} \\
64.8\end{array}$ & $\begin{array}{l}12 \\
34\end{array}$ & 18186.8 & 29.97 & $0.01-1$ & 0.992 & 0.005 & 0.016 & 4.29 \\
\hline Quercetin & - & 300.9 & 150 & $\begin{array}{c}178.8 \\
151.0^{*} \\
120.9\end{array}$ & $\begin{array}{l}14 \\
18 \\
24\end{array}$ & 17670.8 & -23.67 & $0.1-1$ & 0.997 & 0.004 & 0.013 & 9.15 \\
\hline Verbascoside & - & 623.0 & 200 & $\begin{array}{c}461.4 \\
161.0^{*}\end{array}$ & $\begin{array}{l}28 \\
38\end{array}$ & 16911.7 & -8.68 & $0.001-7.5$ & 0.993 & 0.002 & 0.005 & 6.96 \\
\hline Diosmetin & + & 301.0 & 160 & $\begin{array}{l}285.9^{*} \\
257.9 \\
228.7\end{array}$ & $\begin{array}{l}24 \\
36 \\
46\end{array}$ & 107136.9 & 452.09 & $0.001-2.5$ & 0.995 & 0.014 & 0.042 & 10.60 \\
\hline Rutin & - & 609.1 & 200 & $\begin{array}{c}299.9^{*} \\
264.6\end{array}$ & $\begin{array}{l}38 \\
46\end{array}$ & 6303.0 & 0.93 & $0.001-5$ & 0.99994 & 0.000 & 0.001 & 6.60 \\
\hline Luteolin-7-O-glucoside & + & 449.1 & 130 & $287.0^{*}$ & 14 & 122734.2 & 117.66 & $0.001-2.5$ & 0.9998 & 0.003 & 0.010 & 6.80 \\
\hline Tyrosol & - & 136.8 & 100 & $\begin{array}{c}119.0^{*} \\
107.0 \\
105.9 \\
93.0\end{array}$ & $\begin{array}{l}10 \\
10 \\
12 \\
40\end{array}$ & 61.5 & -19.73 & $0.5-5$ & 0.993 & 0.962 & 3.206 & 1.74 \\
\hline
\end{tabular}

Slope and intercept were determined from the calibration curve $(\mathrm{Y}=\mathrm{ax}+\mathrm{b})$. Mode=ionization mode, Frag=fragmentor, $C E=c o l l i s i o n$ energy, $\mathrm{R}^{2}=$ coefficient of determination, $\mathrm{LOD}=$ limit of detection, $\mathrm{LOQ}=$ limit of quantification, $\mathrm{RT}=$ retention time

*quantification transition

min. The column oven temperature was maintained at $30^{\circ} \mathrm{C}$. The sample injection volume was $10 \mu \mathrm{L}$. For MS, the parameters were set as follows: capillary voltage was $4 \mathrm{kV}$ in both positive and negative mode, nozzle voltage $0.5 \mathrm{kV}$, ion source temperature set to $300^{\circ} \mathrm{C}$, gas flow $10 \mathrm{~L} / \mathrm{min}$, nebulizer pressure $241 \mathrm{kPa}$, drying gas temperature $250^{\circ} \mathrm{C}$ and sheath gas flow $11 \mathrm{~L} / \mathrm{min}$.
Nitrogen was used as collision gas and collision energies were from 6 to $50 \mathrm{~V}$ (Table 1).

To establish the calibration curves, reference phenolic compounds were dissolved in methanol and diluted to the appropriate concentrations. For quantitative analysis, 15 different concentrations were made for each standard and the linearity 
ranges of their calibration curves are shown in Table 1. All standard samples were injected in triplicates. Calibration curves were constructed using linear regression and were not forced to pass through zero. Also, a 1/x statistical weight was applied to obtain the most reliable calibration curves for all the phenolics. The calibration curve ranges were obtained based on the linearity of the responses of each individual metabolite (Table 1). The limit of detection (LOD) and limit of quantification (LOQ) were determined using quantifier transition according to the guidelines of the International Council on Harmonization (ICH) (9).

\section{Statistical data processing and design of the experiment}

Statistical data processing was performed using Design-Expert software v. 10.0.3.1 (10). A specialized form of response surface methodology in Design-Expert software was used for designing the mixture formulation. This method established the surface model, estimated all desired components in the mixture and optimized the ratios of individual olive leaf varieties in the final mixture according to the targeted phenolic components. Olive leaf mixtures were designed according to the proposed ratio for each olive leaf cultivar given in the experimental design. Mixtures were prepared with the same optimized extraction method that was used for the characterization of single olive leaf cultivar.

\section{RESULTS AND DISCUSSION}

The phenolic compositions of olive leaf infusions from three olive varieties were analysed using an LC-QQQ method. Firstly, maceration time and temperature were optimized to obtain the highest mass fraction of phenols and to retain the maximum possible diversity of phenolic composition in the olive leaf infusions. Three different temperatures $\left(80,90\right.$ and $\left.100^{\circ} \mathrm{C}\right)$ and five different maceration times $(1,5,10,15$ and $20 \mathrm{~min}$ ) were evaluated in the preparation of olive leaf infusions from the cultivar Istarska bjelica. Total phenolic content was determined by summing up the mass fractions of all detected individual polyphenols. The results revealed that temperature and time had an evident effect on the mass fraction of extracted phenols (Fig. 1). The highest mass fraction was obtained after 15 min of maceration with the starting water temperature of $100^{\circ} \mathrm{C}$.

Optimal maceration parameters were applied for the preparation of infusions with the other two leaf varieties: Leccino and Buža. The LC-QQQ analysis of olive leaf infusions revealed different phenolic compositions of each analysed olive leaf cultivar. The infusion of Istarska bjelica olive leaves had the highest total phenolic mass fraction on dry mass basis $(3.9 \mathrm{mg} / \mathrm{g})$, with oleuropein as the most dominant compound, followed by verbascoside, rutin, luteolin-7-O-glucoside and 3-hydroxytyrosol (Fig. 2). In all tested samples, oleuropein was the major phenolic compound. However, its mass fraction, as well as the presence of other phenolic compounds, was different in each olive leaf cultivar. Leccino and Buža had lower mass fractions of total phenolics than Istarska bjelica, but showed a greater variety in the number of unique phenolic compounds. Interestingly, olive

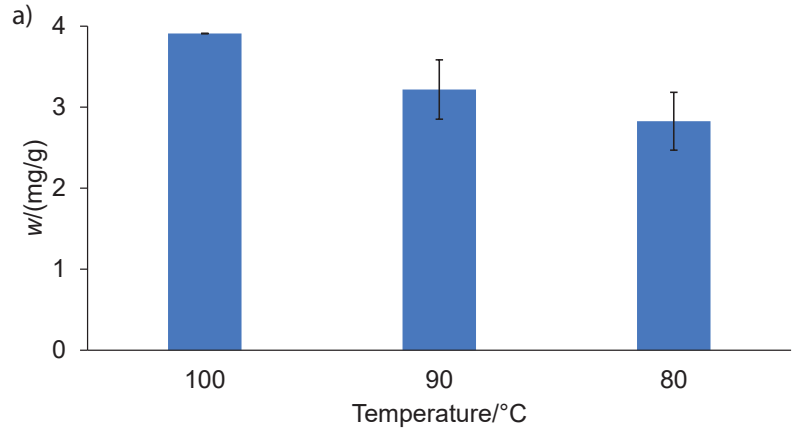

b)

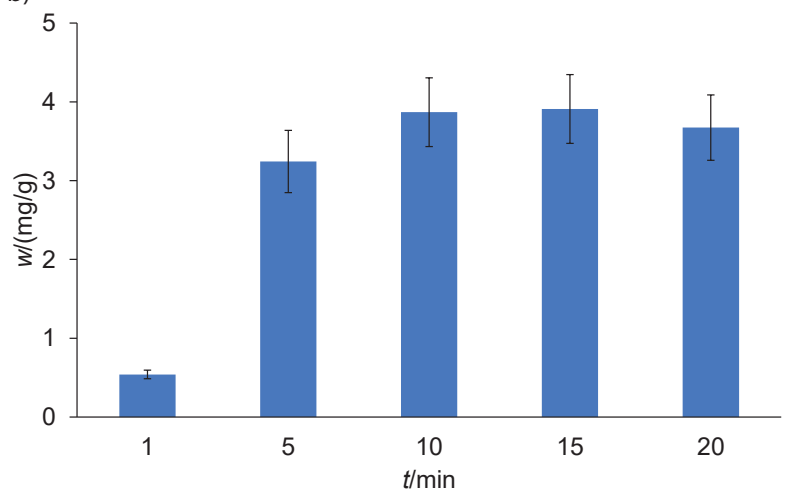

Fig. 1. Total phenolic mass fractions (calculated as the sum of all analysed phenols; mean value \pm standard deviation (S.D.)) in the infusions of Istarska bjelica olive leaves after extraction at: a) different temperatures, and b) different times of maceration

leaf infusions from Buža had two specific phenolic compounds, namely quercetin and diosmetin, that were not detected in the infusions produced from the other leaves.

Phenolic fractions obtained by using the optimized method for infusion preparation were compared with the ones in the extracts of olive leaves produced with organic solvent (methanol). In the organic extraction-based procedure, a high yield of phenolic compounds was achieved. Additionally, extraction was enhanced by treating the infusion with ultrasound for 10 min (which was shown to be the longest ultrasound exposure time for high phenolic yield without causing phenol degradation). In Fig. 3 water-based and organic solvent-based extractions are compared. Fig. 3a shows total mass fractions of phenolic compounds extracted from three different olive leaf varieties using these two methods. Although methanol-based extraction provided higher phenolic mass fractions in the final product, water-based extraction provided rather good results, containing on average $41.83 \%$ of the total phenolics found in the extracts produced with methanol extraction. Furthermore, Fig. 3b shows the mass fractions of the two main compounds quantified in olive leaves, rutin and oleuropein, after the two different extraction procedures. The results show that water-based extraction yielded extracts with higher rutin mass fractions in all cases regardless of the olive cultivar. This was expected, as rutin is a hydrophilic molecule with a $\log P=-0.87$ (11), where $P$ is a partition coefficient. In contrast, oleuropein mass fractions were higher in all methanol extracts, confirming the 

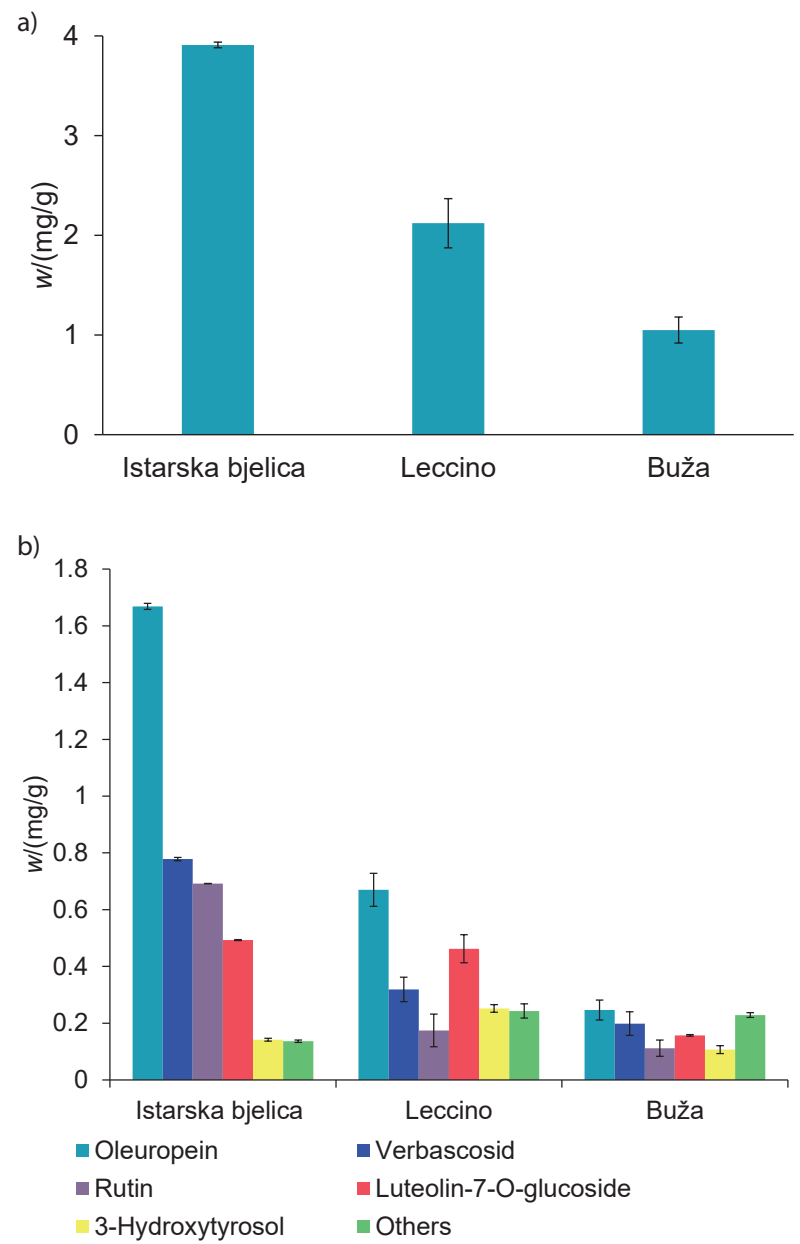

Fig. 2. Mass fractions of: a) total phenols (calculated as the sum of all analysed phenols; mean value \pm S.D.), and b) six main phenols (mean value \pm S.D.) in infusions of Istarska bjelica, Leccino and Buža olive leaves

lipophilicity of these molecules $(\log P=0.11)(11)$. These results support the use of the herein presented method for infusion production, as they reveal that this method provides good phenolic yield and can be used to extract large amounts of hydrophilic compounds.

Based on these findings, we designed formulations for three different functional olive leaf infusions by using mixture design in Design-Expert software (10). The idea was to prepare infusions with different biological activities: mix 1 with a potential positive effect on cardiovascular system, mix 2 with a potential positive effect on reducing diabetes mellitus risk, and mix 3 with a potential antimicrobial activity (Fig. 4). The three olive varieties (Istarska bjelica, Leccino and Buža) analysed in this study were chosen as components for the formulations, and the desired biological effects were adjusted according to the specific phenolic compounds previously identified as bioactives in certain biological processes.

The first mixture was designed as a functional infusion with positive effects on the cardiovascular system. CVDs have always been among the most common causes of death worldwide.
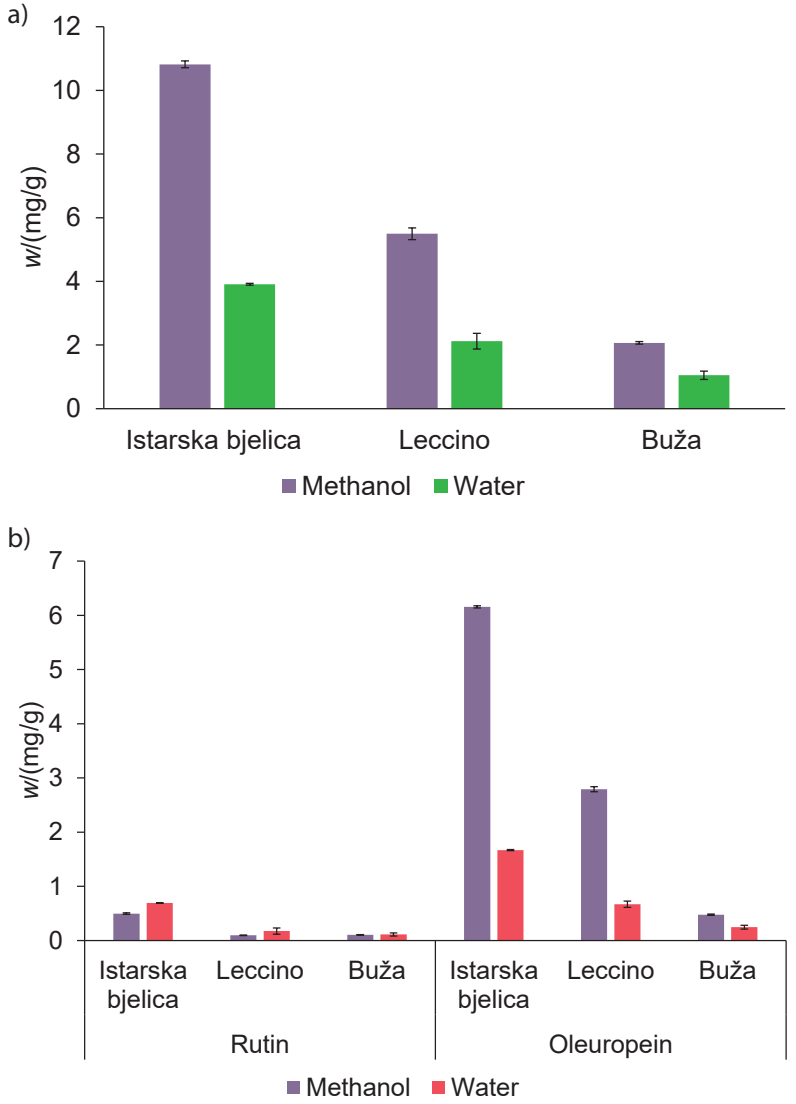

Fig. 3. Mass fractions of: a) total phenols (calculated as the sum of all analysed phenols; mean value \pm S.D.), and $b$ ) of rutin and oleuropein (mean value \pm S.D.) in olive leaf infusions of three different olive cultivars after extractions with methanol and water

They include a number of pathological states that involve cardiac muscles or blood vessels (12). So far, a number of papers have reported that extra virgin olive oil polyphenols have positive effects on CVD. These polyphenols have been found to modulate many CVD risk factors, including inflammation (13), blood pressure (14), and cholesterol levels (15). George et al. (16), for example, analysed the results of 26 such studies and concluded that the intake of olive oil with high polyphenol content aids in the reduction of CVD risks, particularly those related to cholesterol and oxidative stress. Our focus was on atherosclerosis, which is characterized by inflammation in blood vessels $(17,18)$. Indeed, polyphenols can modulate atherosclerosis risk due to their significant antioxidant activity, which is correlated with their structure. Moreover, they modulate cell-signalling cascades by blocking nuclear factor kappa $B$, which is involved in inflammation. Lastly, they have been found to influence the amount of T lymphocytes and monocytes, which bind to the endothelium after blood vessel damage has occurred and which can improve the functioning of endothelia (17). Previous studies examined particular roles of 3-hydroxytyrosol, oleuropein and tyrosol in the prevention of atherosclerosis (18). These studies are the basis for our first design, which was carried out using 3-hydroxytyrosol, ferulic acid, oleuropein, quercetin and tyrosol as responses. Ferulic acid and quercetin 
a)

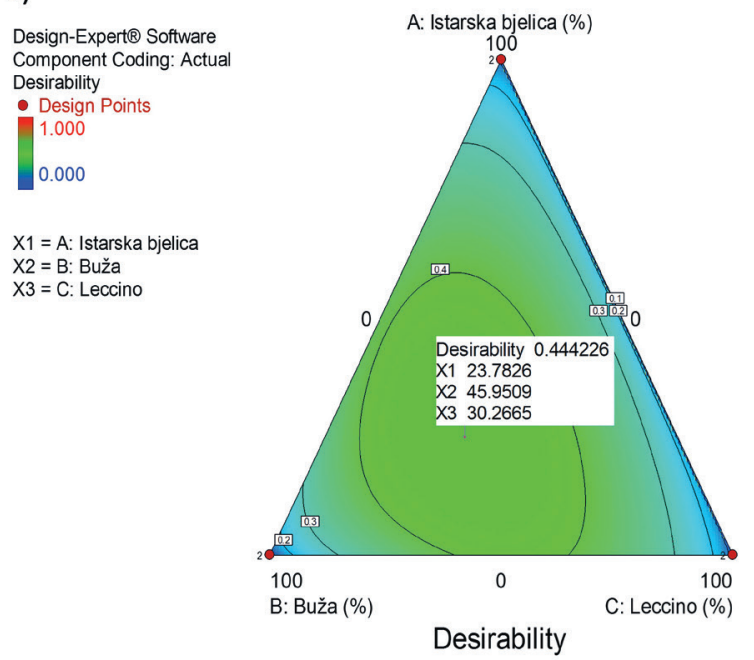

b)

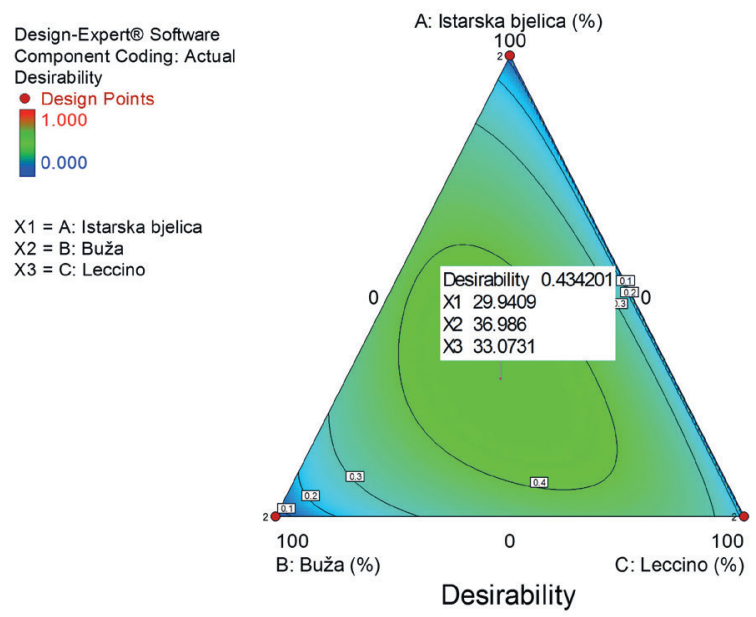

c)

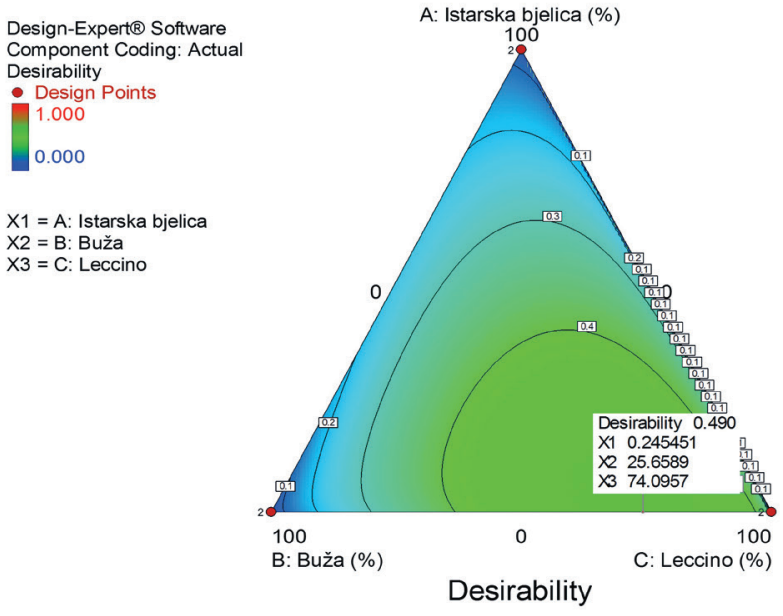

Fig. 4. Contour plots for designed functional infusion mixtures: a) mix 1 with a potential positive effect on cardiovascular system, b) mix 2 with a potential positive effect on reducing diabetes mellitus risk and c) mix 3 with a potential antimicrobial activity were added due to their strong antioxidant and anti-inflammatory properties. Indeed, a clinical trial confirmed that ferulic acid has a high potential to reduce CVD risk factors, as it is believed to improve lipid profiles in blood vessels and to lower oxidative stress level (19). Also, it has been shown that ferulic acid might influence endothelium-dependent myocardial relaxation, suggesting that it benefits the cardiovascular system in terms of cardiac muscles as well as blood vessels (20). Quercetin has been shown to reduce systolic blood pressure but does not seem to have any other known effects in the prevention of CVD (21). Leading bioactives were tyrosol and oleuropein, so they were chosen as the most important responses in the final formulation. Tyrosol was chosen due to its proven inhibitory effect on arachidonic acid production, which lowers the amounts of inflammatory response mediators (22) and inhibits homocysteine-induced endothelial cell adhesion (23). Oleuropein was chosen due to its ability to modulate different processes in atherosclerosis development, such as the expression of endothelial adhesion molecules (24), inhibition of Toll-like receptors (25), and proliferation of smooth muscle cells (26). The final formulation for the first infusion contained $23.8 \%$ Istarska bjelica, 46.0 \% Buža, and 30.2 \% Leccino (Fig. 4a). For tyrosol and oleuropein, as the most important components, the predicted mass fractions in the final mixture were 0.08 and 0.71 $\mathrm{mg} / \mathrm{g}$, respectively (Fig. 5 ).

In 1992, Gonzalez et al. (27) were the first to notice the protective role of olive leaf polyphenols in diabetes, and they opened the area of research on the analyses of metabolism routes of these extracts. Wainstein et al. (28) confirmed that olive leaves can normalise glucose levels in individuals with diabetes. Another trial revealed that olive leaf polyphenol supplementation can improve insulin sensitivity and pancreatic $\beta$-cell secretory capacity (6). In light of this information, we designed the second mixture to be a functional infusion for the reduction of diabetes mellitus risk. The mixture was designed by choosing 2,5-DHBA, 3,4-DHBA, diosmetin, luteolin-7-O-glucoside, rutin, oleuropein, quercetin and 3-hydroxytyrosol as responses. 2,5-DHBA, also called gentisic acid, is one of the sialic acid derivatives that was shown to inhibit the glucose autoxidation-mediated atherogenic modification of low-density lipoproteins (29). 3,4-DHBA was shown to have a high potential for diabetes control. For example, Harini and Pugalendi (30) compared the antihyperglycemic effects of 3,4-DHBA with the known drug glibenclamide, and the results obtained for both compounds were highly comparable. Diosmetin was previously described as a hypoglycemic agent from the plant Anoda cristata (31), so we included it as a response in our research. Luteolin-7-O-glucoside was also chosen as it improves blood glucose, $\mathrm{HbA1c}$, insulin and homeostatic model assessment of insulin resistance (HOMR-IR) levels (32). Moreover, rutin lowers the plasma glucose levels in diabetes-induced mice, stimulates glucose uptake, protects pancreatic cells, and activates liver enzymes that are involved in gluconeogenesis and other metabolic pathways associated with lipids $(33,34)$. Oleuropein was confirmed as a desired polyphenol of high importance in 
a)

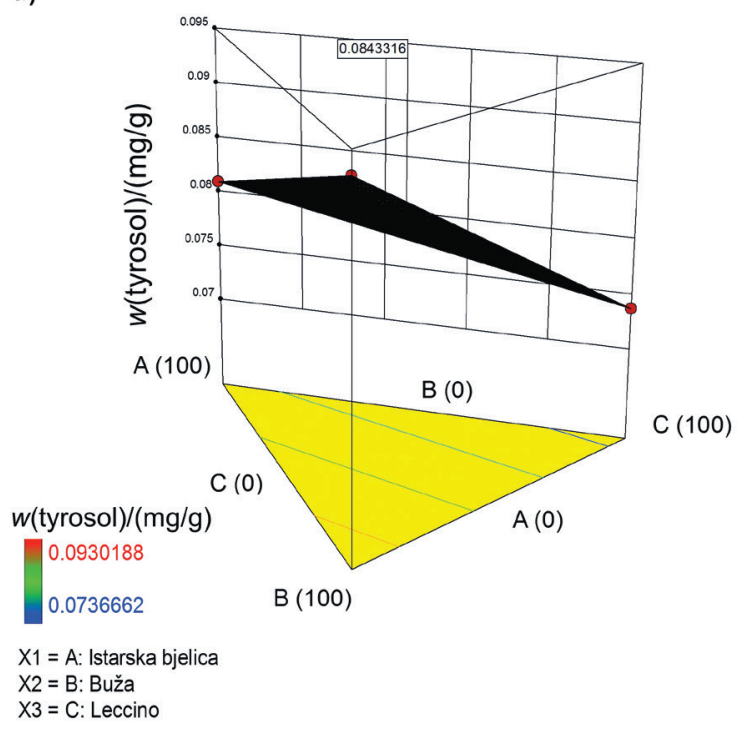

b)

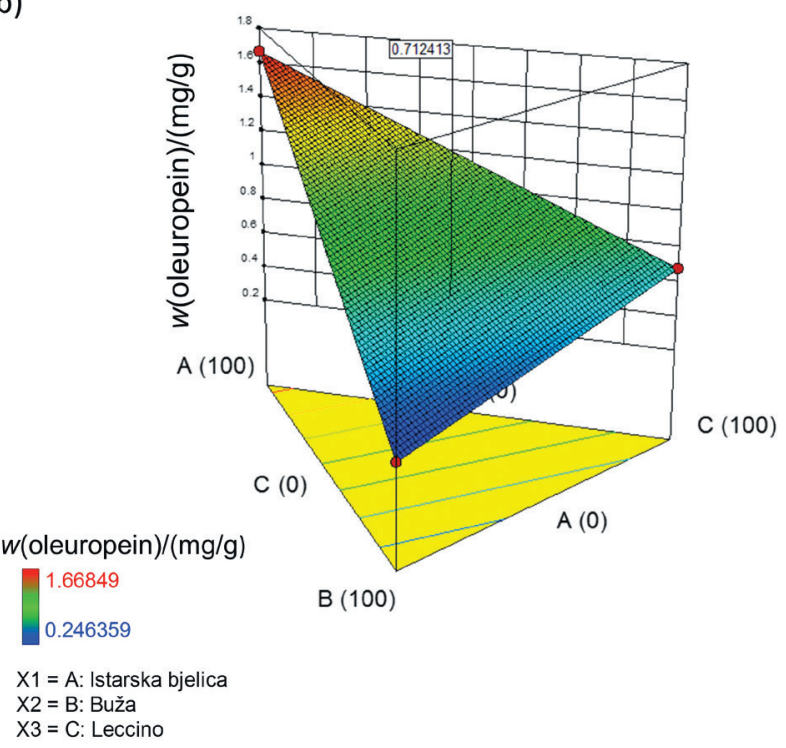

Fig. 5. 3D response surface plots illustrating the effect of olive cultivar in olive leaf infusion mixtures on: a) tyrosol and b) oleuropein mass fractions. Tyrosol and oleuropein were chosen as the most important compounds in mix 1 with possible positive effects on the cardiovascular system. Values in squares are the predicted mass fractions of tyrosol and oleuropein in mix 1

this mixture as per previous reports in which it was shown that, along with 3-hydroxytyrosol, it might exert a significant hypoglycaemic effect. Quercetin can inhibit cytokine-induced cell damage of pancreatic $\beta$-cells (35), which was also confirmed in in vivo studies on rats with induced diabetes (36). Lastly, 3-hydroxytyrosol was chosen as the most important component in the final mixture since it was previously proposed as a pharmaceutical product for metabolic syndrome treatment. Indeed, its role in the downregulation of the sterol regulatory element-binding transcription factor $1 \mathrm{c} /$ fatty acid synthase
(SREBP-1c/FAS) pathway is essential for this purpose (37). Furthermore, 3-hydroxytyrosol demonstrated usefulness in a number of pathological states that occur with diabetes mellitus (38). For example, it has been shown that 3-hydroxytyrosol can be neuroprotective (39). Therefore, the proposed mixture for the second infusion, which was designed to have a positive effect on the reduction of diabetes mellitus risk, was $29.9 \%$ Istarska bjelica, 37.0\% Buža, and 33.1 \% Leccino (Fig. 4b). The predicted mass fractions of 3-hydroxytyrosol and oleuropein in the mixture were 0.17 and $0.81 \mathrm{mg} / \mathrm{g}$, respectively (Fig. 6). a)

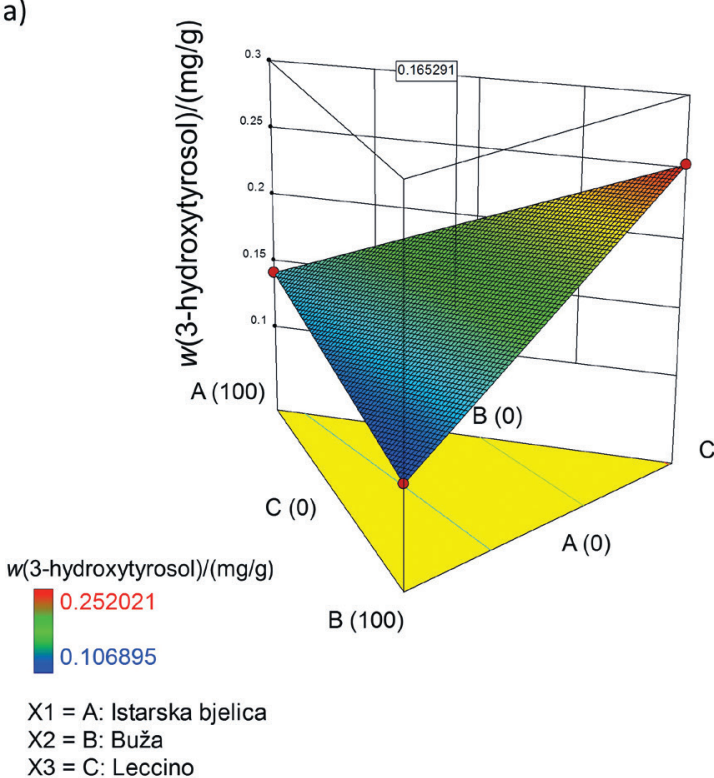

b)

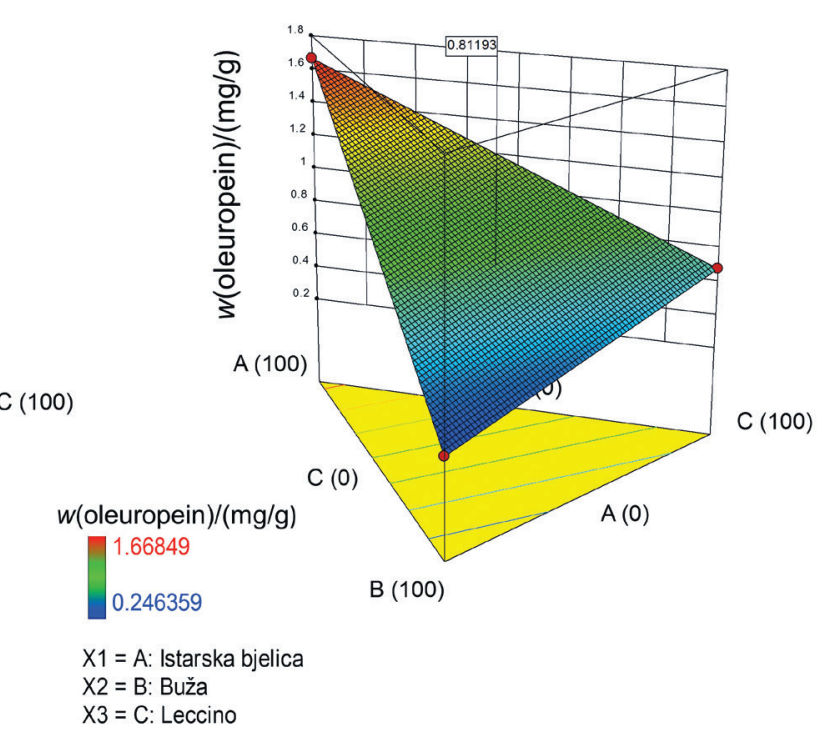

Fig. 6. 3D response surface plots illustrating the effect of olive cultivar in olive leaf mixtures on: a) 3-hydroxytyrosol, and b) oleuropein mass fractions. 3-Hydroxytyrosol and oleuropein were chosen as the most important responses in mix 2 with possible effect on reducing diabetes mellitus risk. Values in squares are the predicted mass fractions of 3-hydroxytyrosol and oleuropein in mix 2 
In addition to their beneficial effects on human health, polyphenols can be exploited in the food industry as antimicrobial ingredients. For example, olive leaf extracts are a natural preservative $(40,41)$. Liu et al. $(42)$ reported that olive leaf extracts can inhibit the growth of Listeria monocytogenes,
Escherichia coli 0157:H7, and Salmonella Enteritidis; they also proposed $62.5 \mathrm{mg} / \mathrm{mL}$ as an effective antibacterial concentration. Therefore, the third mixture investigated in the present study was an antimicrobial mix ture with preferable responses apigenin-7-O-glucoside, caffeic acid, naringenin,

Table 2. Mass fractions of phenolic compounds in three functional olive oil infusions obtained by use of mixture design in Design-Expert software (10)

\begin{tabular}{|c|c|c|c|}
\hline \multirow{2}{*}{ Phenolic compound } & Mix 1 & $\operatorname{Mix} 2$ & Mix 3 \\
\hline & \multicolumn{3}{|c|}{$w /(\mathrm{mg} / \mathrm{g})$} \\
\hline 2,5-DHBA & $0.0007 \pm 0.0001$ & $\begin{array}{c}0.0032 \pm 0.0002 \\
(0.0018) \\
\Delta w=0.0014\end{array}$ & $0.0022 \pm 0.0004$ \\
\hline 3,4-DHBA & $0.0118 \pm 0.0002$ & $\begin{array}{c}0.0402 \pm 0.0009 \\
(0.0448) \\
\Delta w=0.0046\end{array}$ & $0.0236 \pm 0.0006$ \\
\hline 3-Hydroxytyrosol & $\begin{array}{c}0.0850 \pm 0.0072 \\
(0.1592) \\
\Delta w=0.0742\end{array}$ & $\begin{array}{c}0.1219 \pm 0.0002 \\
(0.1653) \\
\Delta w=0.0434\end{array}$ & $0.1399 \pm 0.0076$ \\
\hline Apigenin & $0.0006 \pm 0.0001$ & $0.0007 \pm 0.0000$ & $0.0010 \pm 0.0003$ \\
\hline Apigenin-7-O-glucoside & $0.0235 \pm 0.0000$ & $0.0197 \pm 0.0004$ & $\begin{array}{c}0.0380 \pm 0.0005 \\
(0.0456) \\
\Delta w=0.0076\end{array}$ \\
\hline Caffeic acid & $0.0015 \pm 0.0001$ & $0.0016 \pm 0.0003$ & $\begin{array}{c}0.0022 \pm 0.0001 \\
(0.0032) \\
\Delta w=0.0012\end{array}$ \\
\hline Chrysin & $0.0003 \pm 0.0001$ & $0.0006 \pm 0.0003$ & $0.0003 \pm 0.0001$ \\
\hline Ferulic acid & $\begin{array}{c}0.0029 \pm 0.0008 \\
(0.0152) \\
\Delta w=0.0123\end{array}$ & $0.0048 \pm 0.0016$ & $0.0064 \pm 0.0003$ \\
\hline Gallic acid & $0.0007 \pm 0.0001$ & $0.0067 \pm 0.0005$ & $0.0009 \pm 0.0001$ \\
\hline Luteolin & $0.0031 \pm 0.0001$ & $0.0038 \pm 0.0000$ & $0.0052 \pm 0.0000$ \\
\hline Naringenin & $0.0002 \pm 0.0000$ & $0.0003 \pm 0.0000$ & $\begin{array}{c}0.0004 \pm 0.0000 \\
(0.0006) \\
\Delta w=0.0002\end{array}$ \\
\hline Oleuropein & $\begin{array}{c}1.1554 \pm 0.0034 \\
(0.7124) \\
\Delta w=0.4430\end{array}$ & $\begin{array}{c}0.8198 \pm 0.0032 \\
(0.8118) \\
\Delta w=0.0080\end{array}$ & $0.7640 \pm 0.0261$ \\
\hline$p$-coumaric acid & $0.0042 \pm 0.0004$ & $0.0040 \pm 0.0006$ & $\begin{array}{c}0.0062 \pm 0.0006 \\
(0.0175) \\
\Delta w=0.0113\end{array}$ \\
\hline$p \mathrm{HBA}$ & $0.0022 \pm 0.0002$ & $0.0046 \pm 0.0000$ & $\begin{array}{c}0.0040 \pm 0.0000 \\
(0.0115) \\
\Delta w=0.0075\end{array}$ \\
\hline Quercetin & $\begin{array}{c}0.0046 \pm 0.0003 \\
(0.0002) \\
\Delta w=0.0044\end{array}$ & $\begin{array}{c}0.0066 \pm 0.0003 \\
(0.0002) \\
\Delta w=0.0064\end{array}$ & $\begin{array}{c}0.0078 \pm 0.0008 \\
(0.0001) \\
\Delta w=0.0077\end{array}$ \\
\hline Verbascoside & $0.6719 \pm 0.0171$ & $0.4639 \pm 0.0136$ & $\begin{array}{c}0.4918 \pm 0.0034 \\
(0.2893) \\
\Delta w=0.2025\end{array}$ \\
\hline Diosmetin & $0.0000 \pm 0.0000$ & $\begin{array}{c}0.0001 \pm 0.0001 \\
(0.0006) \\
\Delta w=0.0005\end{array}$ & $0.0001 \pm 0.0000$ \\
\hline Rutin & $0.5070 \pm 0.0018$ & $\begin{array}{c}0.2366 \pm 0.0025 \\
(0.3061) \\
\Delta w=0.0695\end{array}$ & $0.1553 \pm 0.0010$ \\
\hline Luteolin-7-O-glucoside & $0.3846 \pm 0.0132$ & $\begin{array}{c}0.2330 \pm 0.0044 \\
(0.3586) \\
\Delta w=0.1256\end{array}$ & $0.2786 \pm 0.0049$ \\
\hline Tyrosol & $\begin{array}{c}0.0602 \pm 0.0049 \\
(0.0843) \\
\Delta w=0.0241\end{array}$ & $0.0839 \pm 0.0019$ & $0.0770 \pm 0.0047$ \\
\hline
\end{tabular}

Phenolic mass fractions are expressed as mean value \pm standard deviation $(N=2)$ compared to the calculated mass fractions (given in parentheses). $\Delta w=$ mass fraction difference between the calculated values and the values measured by LC-QQQ for given individual phenolic compounds 
p-coumaric acid, $p \mathrm{HBA}$, quercetin and verbascoside. These bioactives have a strong antimicrobial effect. Apigenin-7-O-glucoside has strong antifungal properties and may induce damage to the fungal plasma membrane while inhibiting reactive oxidative species as the main mechanism of antifungal action in 14 different isolates of Candida spp. (43). The same research showed that the antimicrobial activity of apigenin-7-O-glucoside is stronger than that of apigenin. Caffeic acid has also been shown to potentially prevent Candida spp. growth, especially when applied in synergy with other antibiotics (44). In the study of Rauha et al. (45), naringenin was effective in preventing Staphylococcus aureus growth. Moreover, $p$-coumaric acid demonstrated strong antibacterial activity (46). For example, p-coumaric acid efficiently inhibited bacterial growth by increasing the outer and plasma membrane permeability, thus diminishing their barrier function. Additionally, the findings of the same authors show that hydroxycinnamic acid can block bacterial replication, transcription and expression. $p H B A$ was also found to be a strong antibacterial agent (31). Lastly, quercetin and verbascoside were added to this third mixture due to their strong bacteriostatic properties, which have been proven mainly through research on Staphylococcus aureus and Escherichia coli $(47,48)$. The third infusion mixture was expected to exert antimicrobial activity with a wide range of actions. The proposed mixture design for this infusion is shown in Fig. 4c.

Infusions were prepared from designed mixtures 1,2 and 3 by using the optimized water-based method, and their phenolic content was analysed. The quantitative results of each individual polyphenol content in the designed infusion mixtures are presented in Table 2. For the majority of target phenolic compounds, predicted mass fractions were consistent with or were within the acceptable range of those measured by the MS.

It should be noted that the employed design of functional products was based on previous scientific data on the biological activity of the chosen phenolic compounds. As a result, the designed functional products may, theoretically, not induce the expected biological effects. Therefore, the biological evaluation of each infusion effect should be done. The findings of such experiments would provide comprehensive data on the true beneficial effects of these mixtures in the contexts of different health problems. Additionally, it would be interesting to design a new olive leaf infusion with beneficial effects on neurodegeneration processes. Recently, many scientific studies have focused on the effects of olive polyphenols on age-associated neurodegeneration processes (5). For example, it has been shown that these constituents interfere with the aggregation of peptides and proteins in the development of Alzheimer's and Parkinson's diseases and that they may protect cells against the age-associated functional derangement.

Finally, as polyphenols can influence the cytochrome family of enzymes and can even act antagonistically upon each other (49), additional research on these interactions is necessary for the safe preparation of an optimal functional olive leaf infusion.

\section{CONCLUSIONS}

We have presented a unique approach for the development of three new functional infusions based on different mixtures of leaves of three olive cultivars, Istarska bjelica, Buža and Leccino. First, we determined the optimal conditions for infusion preparation: maceration for $15 \mathrm{~min}$ in water heated to $100^{\circ} \mathrm{C}$. Phenolic characterization of the extracts showed that Istarska bjelica had the highest mass fraction of total phenols, with oleuropein as its dominant phenol. Although with the lowest mass fraction of phenols, Buža had the most diverse phenolic composition. Leccino had moderate mass fractions of all phenols, but the highest mass fraction of 3-hydroxytyrosol among the analysed cultivars. These data were statistically processed to design infusions that have specific biological effects. The first mixture was optimized to protect against cardiovascular diseases and it consisted of $23.8 \%$ Istarska bjelica, 46.0 \% Buža and $30.3 \%$ Leccino cultivars. The second mixture intended for the reduction of diabetes mellitus risk was composed of $29.9 \%$ Istarska bjelica, $37.0 \%$ Buža and 33.1 $\%$ Leccino. The third infusion mixture was designed to exert antimicrobial activity, with optimal composition of $0.2 \%$ Istarska bjelica, 25.7 \% Buža and 74.1 \% Leccino. The approach presented in this paper can be further used to develop more functional products based on olive leaves. Also, new olive leaf cultivars with unique phenolic compositions could be added to enrich the infusions with other compounds that could possibly contribute to different biological activities.

\section{ACKNOWLEDGEMENTS}

We greatly appreciate the permission to use the equipment owned by the University of Rijeka, Rijeka, Croatia, within the project "Research Infrastructure for Campus-Based Laboratories at University of Rijeka".

\section{FUNDING}

This work was supported by the European Regional Development Fund (ERDF) and the University of Rijeka, Rijeka, Croatia, research support 13.11.1.1.11 and uniri-biomed-18-133.

\section{CONFLICT OF INTEREST}

The authors have no conflict of interest to declare.

\section{ORCID IDs}

Ž. Peršurić (i) https://orcid.org/0000-0003-0045-1269

L. Saftić (1) https://orcid.org/0000-0001-5637-4659

D. Klisović (i) https://orcid.org/0000-0002-9580-8007

S. Kraljević Pavelić (D) https://orcid.org/0000-0003-0491-673X

\section{REFERENCES}

1. Marín L, Miguélez EM, Villar CJ, Lombó F. Bioavailability of dietary polyphenols and gut microbiota metabolism: Antimicrobial properties. Biomed Res Int. 2015;2015:Article ID 905215.

https://doi.org/10.1155/2015/905215 
2. Ghanbari R, Anwar F, Alkharfy KM, Gilani AH, Saari N. Valuable nutrients and functional bioactives in different parts of olive (Olea europaea L.) - A review. Int J Mol Sci. 2012;13(3):3291-340. https://doi.org/10.3390/ijms13033291

3. Silva S, Gomes L, Leitão F, Coelho AV, Boas LV. Phenolic compounds and antioxidant activity of Olea europaea L. fruits and leaves. Food Sci Technol Int. 2006;12(5):385-95. https://doi.org/10.1177/1082013206070166

4. Lockyer S, Rowland I, Spencer JPE, Yaqoob P, Stonehouse W. Impact of phenolic-rich olive leaf extract on blood pressure, plasma lipids and inflammatory markers: A randomised controlled trial. Eur J Nutr. 2017;56(4):1421-32. https://doi.org/10.1007/s00394-016-1188-y

5. Casamenti F, Stefani M. Olive polyphenols: New promising agents to combat aging-associated neurodegeneration. Expert Rev Neurother. 2017;17(4):345-58.

https://doi.org/10.1080/14737175.2017.1245617

6. De Bock M, Derraik JGB, Brennan CM, Biggs JB, Morgan PE, Hodgkinson SC, et al. Olive (Olea europaea L.) leaf polyphenols improve insulin sensitivity in middle-aged overweight men: A randomized, placebo-controlled, crossover trial. PLoS One. 2013;8(3):e57622.

https://doi.org/10.1371/journal.pone.0057622

7. Kovačić I, Bilić J, Dudaš S, Poljuha D. Phenolic content and antioxidant capacity of Istrian olive leaf infusions. Poljoprivreda. 2017;23(2):38-45.

https://doi.org/10.18047/poljo.23.2.6

8. Hoffmann E de, Stroobant V. Mass spectrometry: Principles and applications. London, UK: John Wiley \& Sons; 2007.

9. Validation of analytical procedures: Text and methodology Q2(R1). ICH harmonised tripartite guideline. International conference on harmonisation of technical requirements for registration of pharmaceuticals for human use. The International Council for Harmonisation of Technical Requirements for Pharmaceuticals for Human Use (ICH), Geneva, Switzerland; 2005. Available from: https://www.ich.org/fileadmin/ Public_Web_Site/ICH_Products/Guidelines/Quality/Q2 R1/Step4/Q2_R1_Guideline.pdf.

10. Design-Expert v.10.0.3.1, Stat-Ease, Inc., Minneapolis, MN, USA; 2016. Available from: https://www.statease.com/dx11.html.

11. Swain M.chemicalize.org.JChem InfModel.2012;52(2):613-5. https://doi.org/10.1021/ci300046g

12. GazianoT, Reddy KS, Paccaud F, Horton S, Chaturvedi V. Cardiovascular disease. In: Jamison DT, Breman JG, Measham AR, Alleyne G, Claeson M, Evans DB, et al., editors. Disease control priorities in developing countries. New York, NY, USA: Oxford University Press; 2006. pp. 645-63.

13. Beauchamp GK, Keast RSJ, Morel D, Lin J, Pika J, Han Q, et al. Ibuprofen-like activity in extra-virgin olive oil. Nature. 2005;437(7055):45-6.

https://doi.org/10.1038/437045a
14. Perona J, Cañizares J, Montero E, Sánchez-Domínguez JM, Catalá A, Ruiz-Gutiérrez V. Virgin olive oil reduces blood pressure in hypertensive elderly subjects. Clin Nutr. 2004;23(5):1113-21.

https://doi.org/10.1016/j.clnu.2004.02.004

15. Farràs M, Castañer O, Martín-Peláez S, Hernáez Á, Schröder $\mathrm{H}$, Subirana I, et al. Complementary phenol-enriched olive oil improves HDL characteristics in hypercholesterolemic subjects. A randomized, double-blind, crossover, controlled trial. The VOHF study. Mol Nutr Food Res. 2015;59(9):1758-70. https://doi.org/10.1002/mnfr.201500030

16. George ES, Marshall S, Mayr HL, Trakman GL, Tatucu-Babet OA, Lassemillante ACM, et al. The effect of high-polyphenol extra virgin olive oil on cardiovascular risk factors: A systematic review and meta-analysis. Crit Rev Food Sci Nutr. 2018;01-138.

https://doi.org/10.1080/10408398.2018.1470491

17. Tangney CC, Rasmussen HE. Polyphenols, inflammation, and cardiovascular disease. Curr Atheroscler Rep. 2013;15(5):324.

https://doi.org/10.1007/s11883-013-0324-X

18. Souza PAL de, Marcadenti A, Portal VL. Effects of olive oil phenolic compounds on inflammation in the prevention and treatment of coronary artery disease. Nutrients. 2017;9(10).

https://doi.org/10.3390/nu9101087

19. Bumrungpert A, Lilitchan S, Tuntipopipat S, Tirawanchai $\mathrm{N}$, Komindr S. Ferulic acid supplementation improves lipid profiles, oxidative stress, and inflammatory status in hyperlipidemic subjects: A randomized, double-blind, placebo-controlled clinical trial. Nutrients. 2018;10(6).

https://doi.org/10.3390/nu10060713

20. Alam MA, Sernia C, Brown L. Ferulic acid improves cardiovascular and kidney structure and function in hypertensive rats. J Cardiovasc Pharmacol. 2013;61(3):240-9.

https://doi.org/10.1097/FJC.0b013e31827cb600

21. Zahedi M, Ghiasvand R, Feizi A, Asgari G, Darvish L. Does quercetin improve cardiovascular risk factors and inflammatory biomarkers in women with type 2 diabetes: A double-blind randomized controlled clinical trial. Int J Prev Med. 2013;4(7):777-85.

22. Moreno JJ. Effect of olive oil minor components on oxidative stress and arachidonic acid mobilization and metabolism by macrophages RAW 264.7. Free Radic Biol Med. 2003;35(9):1073-81.

23. Manna C, Napoli D, Cacciapuoti G, Porcelli M, Zappia V. Olive oil phenolic compounds inhibit homocysteine-induced endothelial cell adhesion regardless of their different antioxidant activity. J Agric Food Chem. 2009;57(9):3478-82. https://doi.org/10.1021/jf8037659

24. Carluccio MA, Siculella L, Ancora MA, Massaro M, Scoditti $E$, Storelli $C$, et al. Olive oil and red wine antioxidant 
polyphenols inhibit endothelial activation: Antiatherogenic properties of Mediterranean diet phytochemicals. Arterioscler Thromb Vasc Biol. 2003;23(4):622-9.

https://doi.org/10.1161/01.ATV.0000062884.69432.A0

25. Ryu SJ, Choi HS, Yoon KY, Lee OH, Kim KJ, Lee BY. Oleuropein suppresses LPS-induced inflammatory responses in RAW 264.7 cell and zebrafish. J Agric Food Chem. 2015;63(7):2098-105.

https://doi.org/10.1021/jf505894b

26. Abe R, Beckett J, Abe R, Nixon A, Rochier A, Yamashita N, et al. Olive oil polyphenol oleuropein inhibits smooth muscle cell proliferation. Eur J Vasc Endovasc Surg. 2011;41(6):81420.

https://doi.org/10.1016/j.ejvs.2010.12.021

27. Gonzalez M, Zarzuelo A, Gamez M, Utrilla M, Jimenez J, Osuna I. Hypoglycemic activity of olive leaf. Planta Med. 1992;58(06):513-5.

https://doi.org/10.1055/s-2006-961538

28. Wainstein J, Ganz T, Boaz M, Bar Dayan Y, Dolev E, Kerem Z, Madar Z. Olive leaf extract as a hypoglycemic agent in both human diabetic subjects and in rats. J Med Food. 2012;15(7):605-10. https://doi.org/10.1089/jmf.2011.0243

29. Exner M, Hermann M, Hofbauer R, Kapiotis S, Speiser W, Held I, et al. The salicylate metabolite gentisic acid, but not the parent drug, inhibits glucose autoxidation-mediated atherogenic modification of low density lipoprotein. FEBS Lett. 2000;470(1):47-50.

https://doi.org/10.1016/S0014-5793(00)01289-8

30. Harini R, Pugalendi KV. Antihyperglycemic effect of protocatechuic acid on streptozotocin-diabetic rats. J Basic Clin Physiol Pharmacol. 2010;21(1):79-91.

https://doi.org/10.1515/JBCPP.2010.21.1.79

31. Juárez-Reyes K, Brindis F, Medina-Campos ON, Pedraza-Chaverri J, Bye R, Linares E, Mata R. Hypoglycemic, antihyperglycemic, and antioxidant effects of the edible plant Anoda cristata. J Ethnopharmacol. 2015;161:36-45. https://doi.org/10.1016/j.jep.2014.11.052

32. Zang Y, Igarashi K, Li Y. Anti-diabetic effects of luteolin and luteolin-7-O-glucoside on KK-A(y) mice. Biosci Biotechnol Biochem. 2016;80(8):1580-6.

https://doi.org/10.1080/09168451.2015.1116928

33. Prince PSM, Kannan NK. Protective effect of rutin on lipids, lipoproteins, lipid metabolizing enzymes and glycoproteins in streptozotocin-induced diabetic rats. J Pharm Pharmacol. 2006;58(10):1373-83.

https://doi.org/10.1211/jpp.58.10.0011

34. Fernandes AAH, Novelli ELB, Okoshi K, Okoshi MP, Di Muzio BP, Guimarães JFC, Fernandes Junior F. Influence of rutin treatment on biochemical alterations in experimental diabetes. Biomed Pharmacother. 2010;64(3):214-9.

https://doi.org/10.1016/j.biopha.2009.08.007
35. Kim EK, Kwon KB, Song MY, Han MJ, Lee JH, Lee YR, et al. Flavonoids protect against cytokine-induced pancreatic $\beta$-cell damage through suppression of nuclear factor $\mathrm{KB}$ activation. Pancreas. 2007;35(4):e1-9.

https://doi.org/10.1097/mpa.0b013e31811ed0d2

36. Coskun O, Kanter M, Kormaz A, Oter S. Quercetin, a flavonoid antioxidant, prevents and protects streptozotocin-induced oxidative stress and $\beta$-cell damage in rat pancreas. Pharmacol Res. 2005;51(2):117-23.

https://doi.org/10.1016/j.phrs.2004.06.002

37. Cao K, Xu J, Zou X, Li Y, Chen C, Zheng A, et al. Hydroxytyrosol prevents diet-induced metabolic syndrome and attenuates mitochondrial abnormalities in obese mice. Free Radic Biol Med. 2014;67:396-407.

https://doi.org/10.1016/j.freeradbiomed.2013.11.029

38. Wani TA, Masoodi FA, Gani A, Baba WN, Rahmanian N, Akhter $\mathrm{R}$, et al. Olive oil and its principal bioactive compound: Hydroxytyrosol - A review of the recent literature. Trends Food Sci Technol. 2018;77:77-90. https://doi.org/10.1016/J.TIFS.2018.05.001

39. Reyes JJ, Villanueva B, López-Villodres JA, De La Cruz JP, Romero L, Rodríguez-Pérez MD, et al. Neuroprotective effect of hydroxytyrosol in experimental diabetes mellitus. J Agric Food Chem. 2017;65(22):4378-83.

https://doi.org/10.1021/acs.jafc.6b02945

40. Ahmed AM, Rabii NS, Garbaj AM, Abolghait SK. Antibacterial effect of olive (Olea europaea L.) leaves extract in raw peeled undeveined shrimp (Penaeus semisulcatus). Int J Vet Sci Med. 2014;2(1):53-6.

https://doi.org/10.1016/J.IJVSM.2014.04.002

41. Albertos I, Avena-Bustillos RJ, Martín-Diana AB, Du WX, Rico D, McHugh TH. Antimicrobial olive leaf gelatin films for enhancing the quality of cold-smoked salmon. Food Packaging Shelf Life. 2017;13:49-55.

https://doi.org/10.1016/J.FPSL.2017.07.004

42. Liu Y, McKeever LC, Malik NSA. Assessment of the antimicrobial activity of olive leaf extract against foodborne bacterial pathogens. Front Microbiol. 2017;8:Article No. 113. https://doi.org/10.3389/fmicb.2017.00113

43. Smiljkovic M, Stanisavljevic D, Stojkovic D, Petrovic I, Marjanovic Vicentic J, Popovic J, et al. Apigenin-7-O-glucoside versus apigenin: Insight into the modes of anticandidal and cytotoxic actions. EXCLI J. 2017;16:795-807.

https://doi.org/10.17179/excli2017-300

44. Sardi JDCO, Gullo FP, Freires IA, Pitangui NDS, Segalla MP, Fusco-Almeida AM, et al. Synthesis, antifungal activity of caffeic acid derivative esters, and their synergism with fluconazole and nystatin against Candida spp. Diagn Microbiol Infect Dis. 2016;86(4):387-91.

https://doi.org/10.1016/j.diagmicrobio.2016.08.002

45. Rauha JP, Remes S, Heinonen M, Hopia A, Kähkönen M, Kujala $T$, et al. Antimicrobial effects of Finnish plant extracts 
containing flavonoids and other phenolic compounds. Int J Food Microbiol. 2000;56(1):3-12.

https://doi.org/10.1016/S0168-1605(00)00218-X

46. Lou Z, Wang H, Rao S, Sun J, Ma C, Li J. p-Coumaric acid kills bacteria through dual damage mechanisms. Food Control. 2012;25(2):550-4.

https://doi.org/10.1016/J.FOODCONT.2011.11.022

47. Fazly Bazzaz BS, Khameneh B, Zahedian Ostad MR, Hosseinzadeh $\mathrm{H}$. In vitro evaluation of antibacterial activity of verbascoside, lemon verbena extract and caffeine in combination with gentamicin against drug-resistant Staphylococcus aureus and Escherichia coli clinical isolates. Avicenna J Phytomed. 2018;8(3):246-53.

48. Wang S, Yao J, Zhou B, Yang J, Chaudry MT, Wang M, et al. Bacteriostatic effect of quercetin as an antibiotic alternative in vivo and its antibacterial mechanism in vitro. J Food Prot. 2018;81(1):68-78.

https://doi.org/10.4315/0362-028X.JFP-17-214

49. Basheer L, Kerem Z. Interactions between CYP3A4 and dietary polyphenols. Oxid Med Cell Longev. 2015;2015:Article ID 854015.

https://doi.org/10.1155/2015/854015 